\title{
Experiencias de Aprendizaje en Ingeniería Química: Diseño, montaje y Puesta en Marcha de una Unidad de Destilación a Escala Laboratorio Mediante el Aprendizaje Basado en Problemas
}

\author{
Fernando Vega*, Esmeralda Portillo, Mercedes Cano y Benito Navarrete \\ Universidad de Sevilla, Dpto. de Ingeniería Química y Ambiental, Escuela Técnica Superior de Ingeniería, \\ C/ Camino de los Descubrimientos, s/n. 41092, Sevilla -España (e-mail: fvega1@etsi.us.es). \\ ${ }^{*}$ Autor a quien debe ser dirigida la correspondencia
}

Recibido Jun. 6, 2013; Aceptado Jul. 18, 2013; Versión final recibida Ago. 28, 2013

\begin{abstract}
Resumen
Se ha propuesto y puesto en práctica una nueva metodología de aprendizaje basado en problemas en una asignatura de carácter experimental en la carrera de ingeniería química en la Escuela Técnica Superior de Ingeniería de la Universidad de Sevilla (España). La metodología se basa en el trabajo con grupos reducidos y aprendizaje basado en problemas, proponiendo nuevos desafíos y oportunidades para el desarrollo de competencias de alumnos de ingeniería. Los alumnos han desarrollado tareas de diseño de procesos, selección de alternativas, toma de decisiones, diseño de ingeniería básica y gestión de compras, que han culminado con el montaje y puesta en marcha de una unidad de destilación a escala laboratorio. La metodología ha permitido trabajar todas las competencias adscritas a la asignatura con un alto grado de participación y motivación del alumnado. Por lo tanto, se considera una herramienta atractiva y útil para su incorporación en asignaturas de experimentación en ingeniería química
\end{abstract}

Palabras clave: metodología de aprendizaje, enseñanza en ingeniería, aprendizaje basado en problemas, auto-aprendizaje

\section{Teaching Experiences in Chemical Engineering: Design, Manufacturing and Start-up of a Lab-scale Distillation Unit using Problem-based Learning}

\begin{abstract}
A new problem solving methodology is proposed for a course which is essentially experimental for student of chemical engineer at the Higher Technical Engineering School of the University of Seville in Spain is proposed. The methodology is based on small group work and problem-based learning, proposing new challenges and opportunities to develop skills and competencies in engineering student. The students developed tasks regarding process design, selection of optimal alternative, decision making, basic engineering design and purchase management, which concluded with the assembly and starting-up of a laboratory scales distillation unit. The methodology allowed working all subject competences with high participation and motivation from the students. Therefore, it is considered to be a useful and attractive tool to be incorporated to experimental chemical engineering courses.
\end{abstract}

Keywords: learning methodology, engineering teaching, problem-based learning, self-learning 


\section{INTRODUCCIÓN}

La convergencia hacia el Espacio Europeo de Educación Superior (EEES), en el ámbito de la enseñanza universitaria en España, está exigiendo en los últimos años nuevos retos docentes y metodológicos encaminados a la equidad en la formación universitaria a nivel europeo, dentro del marco del denominado Proceso Bolonia (De la Iglesia, 2012; Mesa et al., 2011; Jiménez et al., 2011). El desarrollo de nuevas metodologías didácticas, sistemas alternativos de evaluación y el diseño de nuevas actividades están suponiendo los retos más exigentes para las universidades españolas en la actualidad (Pérez-Cedrán et al., 2013). En España, la aprobación de la Ley orgánica 4/2007, del 12 de abril permitió definir las bases sobre las que se sustenta la consecución de este objetivo. Además de la reestructuración de los planes de estudios en grados - máster - doctorado, estas bases pueden ser resumidas en tres pilares fundamentales: los nuevos modelos de aprendizaje basados en la adquisición de competencias para su desarrollo en el ejercicio profesional (De la Iglesia, 2011; Ayuga Téllez et al., 2010), la descentralización del profesor en la acción educativa por el fomento del auto-aprendizaje y autogestión por parte del alumno (Jiménez et al. 2011; Rué, 2007) y la transmisión de hábitos y comportamientos que potencien los conocimientos adquiridos (Branda, 2009).

Este nuevo escenario educativo requiere del desarrollo de herramientas de aprendizaje y metodologías docentes que sustituyan a los métodos tradicionales de enseñanza. En este sentido, el trabajo con grupos reducidos (Bogumil y Abad, 2008) y el aprendizaje basado en problemas (ABP) son considerados por los profesionales docentes como dos de los métodos de aprendizaje con mayor potencial, flexible y adaptable a las nuevas necesidades planteadas por el EEES (Benítez y García, 2013; Ribeiro, 2011; Branda, 2009; Rué, 2007; Morales y Fitzgerald, 2004). El aprendizaje basado en problemas se basa en la exposición previa de problemas reales cuyo objetivo es que el alumno sea capaz de resolver durante el periodo de aprendizaje guiado. Para ello, es imprescindible una definición clara y concisa de los objetivos y condiciones de aprendizaje por parte del profesor y la identificación de las necesidades de aprendizaje y de información por parte del alumno. La aplicación de este tipo de métodos de aprendizaje pretende fomentar en el alumnado un papel más activo en el aprendizaje, sustituyendo el rol tradicional del profesor como mero transmisor del conocimiento por una figura que active, asesore y dirija durante el proceso de aprendizaje. El alumno adquiere una mayor libertad sobre su aprendizaje pero, a su vez, una mayor responsabilidad. El profesor debe incentivar y promover inquietudes sobre los alumnos, actuando como un motivador en la acción educativa (Latasa et al., 2012; Jiménez et al., 2011; Ribeiro, 2011).

El objetivo general de este trabajo ha consistido en la puesta en marcha de una metodología docente basada en el trabajo con grupos reducidos y el aprendizaje basado en problemas, que complementen la actual enseñanza teórico-práctica de la materia experimentación en ingeniería química y que satisfaga los retos educativos planteados por el EEES. Esta metodología ha sido aplicada en la asignatura 'Experimentación en planta piloto' de quinto curso de la titulación de Ingeniero Químico y de Ingeniero Industrial durante el curso académico 2012-2013. La asignatura se compone de varios grupos de entre 6 y 8 alumnos, siendo la metodología propuesta en este trabajo aplicada a un único grupo. Dado el carácter troncal de la asignatura, optativa para la rama industrial, y su encuadre en el último curso, hacen de esta propuesta una oportunidad extraordinaria para que los alumnos dispongan de espacio educativo donde aplicar las capacidades adquiridas con anterioridad en el campo de la ingeniería a todos los niveles: diseño, gestión, compras, montaje, planificación, operación, etc.

Dentro de la actividad docente, se ha propuesto el montaje de una unidad a escala laboratorio que reproduzca una de las principales operaciones de separación de la industria química como es la destilación. El desarrollo de las actividades propuestas proporcionará al alumno un conocimiento sobre el desarrollo de proyectos en ingeniería química y optimización de procesos que en la actualidad son esenciales para los especialistas universitarios en este campo, además de complementar la docencia en áreas como control de procesos, gestión de proyectos, diseños de ingeniería y montajes eléctricos. Esta instalación ha requerido la ejecución de tareas en campos tan diversos como la ingeniería química, mecánica o eléctrica, sirviendo de apoyo a la docencia en materias de gran importancia para un profesional de la ingeniería y escasamente tratadas en los estudios de ingeniería química.

Los hitos específicos de este trabajo, marcados como objetivos por parte del profesorado responsable, son los siguientes:

a) Planificar una estrategia de resolución de problemas ingenieriles que faciliten la asimilación de conceptos teóricos previos, la adquisición de habilidades y destrezas enfocadas a su aplicación en el ejercicio profesional y el desarrollo de las actividades planteadas durante el proyecto docente por parte de los alumnos. 
b) Diseñar una unidad de destilación para el estudio de operaciones de transferencia de materia. En concreto, operaciones de destilación de disoluciones bi- y multi-componente.

c) Desarrollar una metodología docente innovadora que se aproxime a las necesidades y recomendaciones planteadas por los nuevos planes de estudio.

d) Interactuar entre alumnos de áreas de ingeniería química, eléctrica y mecánica.

e) Enfrentar al alumno con problemas reales, promoviendo la resolución de los mismos partiendo de sus conocimientos y experiencias previas y fomentando la búsqueda de información técnica, la familiarización con normativa, el auto-aprendizaje y el trabajo en equipo a partir de la motivación en el aprendizaje.

f) Trabajar competencias dirigidas a las habilidades que se demandan en el ámbito laboral para técnicos especialistas en la industria

g) Dar oportunidad al alumno para realizar todas las etapas de desarrollo de proyectos de ingeniería, a pequeña escala, colaborando en todos los estadios del mismo, desde la idea conceptual hasta el montaje y puesta en marcha de la instalación.

h) Resolver problemas a todos los niveles: dirección de proyecto, montaje, gestión de presupuesto, compras, reparto de tareas, responsabilidades, elección de propuestas, toma de decisiones.

\section{METODOLOGÍA}

La metodología docente propuesta se basa en la resolución de un problema de ingeniería (objetivo global del curso) por parte de un grupo compuesto 8 alumnos. El problema planteado como objetivo global, denominado de aquí en adelante 'proyecto de curso', es disgregado en una serie de problemas de menor envergadura planteados en cada sesión (objetivos a corto plazo) y que han sido estratégicamente planteados durante la planificación del proyecto docente por parte del profesor tutor (Tabla 1). Estas tareas permiten dirigir el aprendizaje hacia los objetivos marcados por los planes de estudios e identificados en el EEES como las competencias a adquirir durante la actividad docente y recogidas en el plan docente de la asignatura, por lo que el profesor tutor es considerado en este proyecto como gestor del aprendizaje (Tabla 2).

Tabla 1: Cronograma del proyecto y descripción de las actividades

\begin{tabular}{|c|c|c|c|c|}
\hline Bloque & Sesión & Fecha & Tareas & Trabajo Semanal \\
\hline \multirow[t]{4}{*}{$\begin{array}{l}\text { Bloque I } \\
\text { Ingeniería } \\
\text { Básica }\end{array}$} & 1 & $08 / 10 / 2012$ & $\begin{array}{c}\text { Presentación del Proyecto } \\
\text { Elaboración de Propuestas de Diseño } \\
\text { (grupos de 2) }\end{array}$ & $\begin{array}{c}\text { Entregable } 0 \text { (Diagrama de Procesos } \\
\text { Idea Conceptual y Diagrama de } \\
\text { Procesos) }\end{array}$ \\
\hline & 2 & $15 / 10 / 2012$ & $\begin{array}{l}\text { Elaboración de Propuestas de Diseño } \\
\text { Síntesis del proceso (grupos de } 2 \text { ) }\end{array}$ & Propuestas \\
\hline & 3 & $22 / 10 / 2012$ & $\begin{array}{c}\text { Defensa de Propuestas y Puesta en } \\
\text { Común } \\
\text { Selección de Alternativas } \\
\text { Memoria de Cálculo }\end{array}$ & Lista de Materiales \\
\hline & 4 & $29 / 10 / 2012$ & $\begin{array}{l}\text { Ingeniería Básica } \\
\text { Gestión de Compras }\end{array}$ & $\begin{array}{c}\text { Entregable } 1 \text { (Memoria Técnica + } \\
\text { P\&ID) }\end{array}$ \\
\hline \multirow[t]{4}{*}{$\begin{array}{l}\text { Bloque II } \\
\text { Desarrollo } \\
\text { Ingenieril }\end{array}$} & 5 & $12 / 11 / 2012$ & $\begin{array}{l}\text { Gestión de Compras } \\
\text { Tareas de Montaje }\end{array}$ & $\begin{array}{c}\text { Acta de Seguimiento } \\
\text { Problemas }+ \text { Soluciones }\end{array}$ \\
\hline & 6 & $13 / 11 / 2012$ & $\begin{array}{l}\text { Gestión de Compras } \\
\text { Tareas de Montaje }\end{array}$ & $\begin{array}{c}\text { Acta de Seguimiento } \\
\text { Problemas + Soluciones }\end{array}$ \\
\hline & 7 & $14 / 11 / 2012$ & Tareas de Montaje & $\begin{array}{c}\text { Acta de Seguimiento } \\
\text { Problemas + Soluciones }\end{array}$ \\
\hline & 8 & $15 / 11 / 2012$ & Tareas de Montaje & $\begin{array}{c}\text { Acta de Seguimiento } \\
\text { Entregable } 2\end{array}$ \\
\hline \multirow[t]{2}{*}{$\begin{array}{l}\text { Bloque III } \\
\text { Operación }\end{array}$} & 9 & $10 / 12 / 2012$ & Puesta en Marcha & $\begin{array}{c}\text { Puesta en Marcha (Certificación de la } \\
\text { instalación) }\end{array}$ \\
\hline & 10 & $17 / 12 / 2012$ & Operación & $\begin{array}{l}\text { Entregable 3(Manuales Puesta en } \\
\text { Marcha, Operación, Parada) }\end{array}$ \\
\hline
\end{tabular}


Dado que el alumno está familiarizado con el aprendizaje basado en problemas y ha desarrollado competencias relacionadas con la resolución de problemas, trabajo en equipo e ingeniería de procesos a lo largo de la titulación, esta metodología les brinda la oportunidad de aplicar y desarrollar estas competencias mediante la resolución de un problema real, traducido en la construcción y puesta en marcha de una unidad a escala laboratorio. La asignatura se encuentra en el último curso de la titulación, por lo que conocimientos sobre operaciones de separación, mecánica de fluidos y termodinámica son requeridos, como queda recogido en el plan de estudios. Esta asignatura es impartida en el mismo periodo docente que la asignatura de control y optimización de procesos, por lo que es una buena oportunidad para afianzar conceptos relacionados con dichas materias. Los profesores tienen en cuenta estas necesidades a la hora de la asignación de tareas, complementando mediante explicaciones a lo largo de las sesiones los aspectos en los que los alumnos no disponen de conocimientos suficientes.

El 'proyecto de curso' consiste en el diseño, montaje y puesta en marcha de una unidad de separación por destilación etanol-agua. El proyecto ha sido dividido en tres bloques principales: Ingeniería básica, donde los alumnos tienen como objetivo completar el diseño y la memoria de la instalación, Desarrollo ingenieril, donde los alumnos potenciaron sus destrezas y habilidades en dos líneas principales: gestión de compras y montaje de instalaciones industriales a escala laboratorio, y Operación. En el último bloque se llevó a cabo la puesta en marcha de la instalación y una simulación de práctica donde se la funcionalidad y operatividad de la unidad de destilación. Esta evaluación fue realizada mediante la operación en continuo de la planta por parte del grupo de alumnos durante la última sesión de curso, mediante la cual fue posible definir los intervalos de operación de las variables manipulables del proceso.

La asignatura consta de un total de 10 sesiones presenciales, de tres horas de duración cada una. La dedicación del alumno en horas no lectivas se encuentra comprendida entre 2 y 5 horas semanales, en función de las tareas, entregables y objetivos solicitados. En las fases iniciales del proyecto, las necesidades en horas no lectivas han sido mayores, mientras que en las últimas fases del proyecto, todas las tareas han sido desarrolladas en el laboratorio, centrándose la dedicación en horas no lectivas en la redacción de informes técnicos y entregables.

En la sesión inicial se elije un coordinador, responsable del seguimiento de la actividad, cumplimiento de los objetivos y encargado de recopilar toda la información generada. Posteriormente, la metodología seguida en cada sesión consiste en la definición de las tareas objetivo, la formación de varios grupos de trabajo en función de cada bloque y el reparto de tareas por parte del propio alumnado. Cada grupo de trabajo elige a un responsable, el cual es encargado de informar sobre los avances y problemas surgidos. Estos equipos de trabajo no son independientes, sino que las tareas encomendadas promueven la necesidad de interacción y trabajo en equipo de los distintos grupos para poder resolver problemas surgidos en el transcurso de las sesiones, fomentando el aprendizaje cooperativo y la capacidad de comunicación. Durante las sesiones, los alumnos consultan bibliografía especializada (McCabe et al., 2007; Perry, 2007; Martín, 1994), alumnos de diversas especialidades, el profesorado y personal externo tales como suministradores, comerciales y técnicos montadores de empresas externas para poder realizar de forma exitosa las tareas encomendadas.

Tabla 2: Competencias trabajadas en cada sesión

\begin{tabular}{|c|c|c|}
\hline Sesión & Competencias transversales & Competencias Específicas \\
\hline 1 & $\begin{array}{c}\text { Capacidad de Análisis y Síntesis } \\
\text { Trabajo en Equipo } \\
\text { Capacidad de organizar y Planificar }\end{array}$ & \multirow[t]{4}{*}{ Cognitivas } \\
\hline 2 & Trabajo en Equipo & \\
\hline 3 & $\begin{array}{c}\text { Capacidad para aplicar la teoría a la práctica } \\
\text { Capacidad de crítica y autocrítica } \\
\text { Habilidades para trabajar en grupo }\end{array}$ & \\
\hline 4 & Solidez en los conocimientos básicos de la profesión & \\
\hline 5 & Planificar y dirigir & \\
\hline 6 & Trabajo en Equipo & \\
\hline 7 & Solidez en los conocimientos básicos de la profesión & \\
\hline 8 & Capacidad de Análisis y Síntesis & \\
\hline \multirow{2}{*}{$\begin{array}{c}9 \\
10\end{array}$} & Trabajo en Equipo & \multirow{2}{*}{$\begin{array}{c}\text { Procedimentales / Instrumentales } \\
\text { Actitudinales }\end{array}$} \\
\hline & Comunicación escrita en lengua nativa & \\
\hline
\end{tabular}


La evaluación del trabajo del alumno se realiza mediante una ponderación de las calificaciones obtenidas en los documentos y entregables solicitados, con un peso del $35 \%$, el examen final de la asignatura, $15 \%$, y el trabajo en el laboratorio, $40 \%$. Este último concepto integra las actividades realizadas durante las sesiones en el laboratorio, como participación activa y dinámica del alumno (10\%), propuestas de ideas innovadoras $(20 \%)$, creatividad $(20 \%)$, destreza en la resolución de problemas $(20 \%)$, actitud en el trabajo en equipo (15\%), limpieza y organización en el trabajo diario (5\%), aplicación correcta de conocimientos y conceptos ingenieriles (10\%). Se pretende potenciar e incentivar en mayor medida las aptitudes, destrezas y habilidades en el laboratorio, así como la libertad creativa del alumno.

\section{RESULTADOS}

La aplicación de la nueva metodología docente en la asignatura 'Experimentación en planta piloto' ha cumplido satisfactoriamente con los objetivos previos marcados. Esta herramienta es apta para acometer actividades docentes en el ámbito del EEES. El éxito de la metodología se ha basado en el trabajo de planificación previo realizado por el profesorado y en la gran aceptación con el que ha sido recibido por parte del alumnado, lo que ha posibilitado generar un excelente clima de trabajo con el que ha sido posible culminar todas las tareas y superar los problemas. Se desglosan a continuación los hitos alcanzados en cada uno de los bloques que componen la actividad.

\section{Bloque I}

El bloque inicial es fundamental, dado que marca el desarrollo posterior del resto de tareas. En este sentido, la dedicación en cómputo de horas durante el desarrollo de las actividades de este bloque ha sido la más elevada y el seguimiento por parte de los profesores más exhaustivo.

En la sesión inicial se expuso el objetivo global del proyecto y la programación (cronograma), así como la metodología docente y los entregables requeridos al alumnado. Esta planificación debe ser revisada cada año en función al proyecto de curso propuesto por el profesorado. La sensación inicial de los alumnos se entremezcla entre la motivación por construir a partir de sus conocimientos algo que pueda ser de utilidad y el miedo al desconocimiento por el cómo alcanzar dichos objetivos. Es importante remarcar la necesidad de estructurar una buena organización en este tipo de experiencias: objetivos claros y realistas, cronograma adecuado a las tareas asignadas, selección de roles (coordinador y responsable), sin la cual puede resultar en vano toda la puesta en escena.

Los alumnos necesitaron varias sesiones para adaptarse a la metodología propuesta, durante las cuales se tuvo que potenciar la interacción alumno-profesor hasta adquirir la dinámica del auto-trabajo por parte de los grupos. De este modo se evitó la dispersión de los objetivos y el sobresfuerzo debido a la mala planificación o a la no identificación clara de objetivos. La división en grupos reducidos para la elaboración de propuestas resultó muy efectiva, consiguió hacer partícipes a todos los alumnos al mismo nivel de exigencia y garantizó un alto nivel de conocimiento de todos los elementos de la instalación a todos los integrantes. Los alumnos dispusieron de total libertad para realizar consultas a profesores, técnicos de laboratorio y comerciales sobre sus ideas para llevar a cabo el diseño de la instalación.

Durante las primeras sesiones, la ingeniería conceptual y un diagrama preliminar del proceso fueron obtenidos en la primera sesión, siempre bajo la supervisión del profesorado. Los criterios de diseño básico se recogen en la Tabla 3. Maximizar el grado de control y operatividad de la instalación así como garantizar la integridad estructural y la seguridad durante la operación fueron tenidos en consideración durante las tareas de diseño.

Tabla 3: Principales criterios de diseño

\begin{tabular}{|l|c|}
\hline \multicolumn{1}{|c|}{ Criterios de diseño } & Valores \\
\hline Caudal de alimentación & $3 \mathrm{~L} / \mathrm{h}$ \\
Temperatura de alimentación & Inferior a $100^{\circ} \mathrm{C}$ \\
Composición en etanol del condesado & $95 \% \mathrm{p} / \mathrm{p}$ \\
Presión de operación & 1 bar \\
Caudal de destilado & $>5 \%$ alimentación \\
\hline
\end{tabular}

En las siguientes sesiones, los alumnos de los diferentes grupos propusieron cuatro diseños diferentes, en base a este documento (entregable 0), que fueron sometidos a debate general con la intención de definir el diseño final de la instalación. El adecuado conocimiento de las necesidades de la instalación y el alto grado 
de participación de cada alumno hizo de esta actividad una de las más satisfactorias y enriquecedoras dentro del proyecto. Los alumnos trabajaron de forma eficiente competencias relacionadas principalmente con el trabajo en equipo, defendiendo cada propuesta y convergiendo hacia una solución única en el caso de disparidad de alternativas.

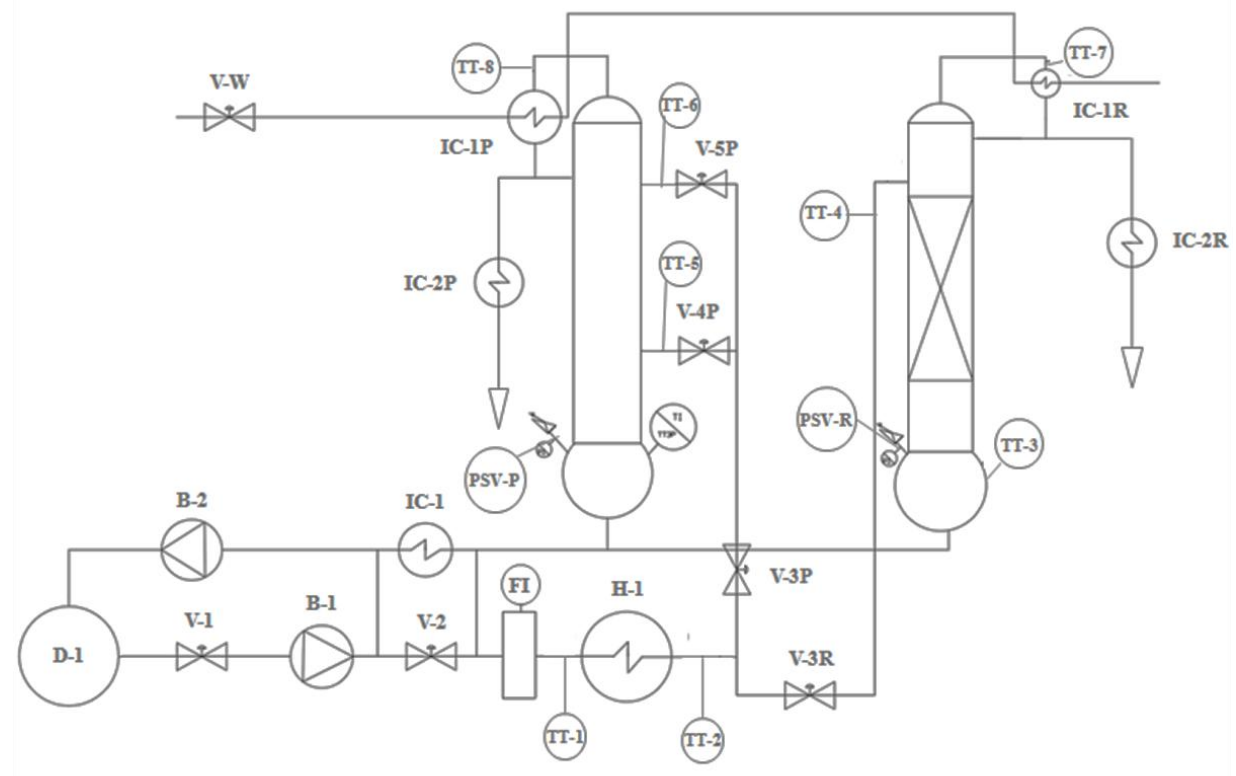

Fig. 1: Diagrama de procesos de la unidad de destilación seleccionada

A partir del diseño integrado entre las propuestas presentadas, los alumnos realizaron la integración energética del proceso haciendo uso de un simulador comercial, con el objetivo de minimizar el consumo del calentador H-1 (Figura1). Una vez definida la configuración final de la instalación, los alumnos comenzaron a realizar tareas relacionadas con trabajos de gabinete, realizando la memoria descriptiva de la instalación, el diagrama de proceso definitivo (Figura 1), la lista de materiales y el cálculo de los equipos de impulsión. Toda la información fue recogida en un documento, que se presentó al inicio de la sesión 5 . El documento fue revisado y devuelto a los alumnos con los comentarios y apreciaciones de los profesores en las dos semanas posteriores a su entrega.

Los principales problemas encontrados durante las sesiones iniciales han sido principalmente la dispersión de los alumnos en algunas de las tareas encomendadas debido a la falta de concreción en la definición de objetivos y el desconocimiento inicial de las herramientas de consulta disponibles para llevarlas a cabo: técnicos, profesorado, profesionales externos. El profesor debe prestar atención a estas circunstancias para identificar si se están realizando tareas no productivas, reconducir a los alumnos en el momento adecuado dentro del proceso de aprendizaje y guiarlos hacia actuaciones eficaces en relación al cumplimiento de los objetivos docentes.

\section{Bloque II}

El bloque II se centró en la gestión de compras y el montaje de la instalación. Es importante destacar la amplia experiencia del departamento de Ingeniería Química y Ambiental de la Escuela Técnica Superior de Ingeniería de Sevilla en el desarrollo de montajes de experiencias de laboratorio y plantas piloto de investigación propias, que posteriormente son integradas en las actividades que componen las asignaturas de prácticas que imparte el departamento. El material requerido para la instalación de la unidad de destilación se obtuvo por dos vías diferentes: material disponible en el laboratorio (40\%) y compras a proveedores (60\%). El trabajo en las sesiones iniciales de este bloque consistió en el acopio de los materiales disponibles y en la identificación del material a adquirir. Durante el desarrollo de las tareas de montaje, el grupo inicial de 8 personas se dividió en dos sub-grupos de 4 alumnos cada uno, encargados del comienzo de las actividades de montaje y de la gestión de compras. Estos grupos de trabajo se intercambiaron tareas en cada sesión. Para ello, se fomentó la interacción entre los alumnos con la intención de facilitar el intercambio de información sobre el estado actual de la actividad y las necesidades inmediatas de cada tarea. Con ello se consiguió la participación de todos los alumnos en ambas actividades. Las tareas llevadas a cabo han permitido a los alumnos adquirir nuevas destrezas y habilidades en diversas áreas de la ingeniería (Tabla 4). Las actividades de montaje finalizaron con entrega de la instalación y del entregable 2, donde incluyeron todas las tareas de montaje realizadas así como una recopilación del histórico de actividades realizadas durante este bloque. 
Tabla 4: Destrezas y habilidades trabajadas durante el desarrollo del Bloque 2

\begin{tabular}{|c|l|}
\hline Área de Trabajo & \multicolumn{1}{c|}{ Destrezas / Habilidades } \\
\hline \multirow{3}{*}{ Ingeniería de Procesos } & $\begin{array}{l}\text { Elaboración e interpretación de diagramas de proceso (P\&ID) } \\
\text { Elaboración e interpretación de isométricos } \\
\text { Integración energética de procesos } \\
\text { Diseño de lazos de control } \\
\text { Selección de equipos e instrumentación } \\
\text { Búsqueda de soluciones técnicas viables en base a los medios disponibles }\end{array}$ \\
\hline \multirow{2}{*}{ Ingeniería Eléctrica } & $\begin{array}{l}\text { Interpretación de esquemas eléctricos } \\
\text { Cableado de equipos e instrumentación en baja tensión (monofásicos) } \\
\text { Selección de protecciones eléctricas } \\
\text { Tratamiento de señales analógicas }\end{array}$ \\
\hline Ingeniería Mecánica & $\begin{array}{l}\text { Conexionado de equipos e instrumentación a proceso } \\
\text { Trabajos de soldadura }\end{array}$ \\
\hline
\end{tabular}

Los principales problemas encontrados durante la ejecución de este bloque radicaron en la puesta en práctica de diferentes habilidades de montaje, que fueron guiadas adecuadamente por los técnicos de laboratorio. Para la ejecución de los trabajos se adoptaron todas las medidas de prevención de riesgos necesarias con objeto de cumplir la normativa vigente en materia de seguridad, siguiendo el plan de seguridad de los laboratorios. No se produjo ningún accidente o incidencia en materia de seguridad durante el periodo de montaje de la instalación.

\section{Bloque III}

Las últimas dos sesiones se dedicaron a la puesta en marcha y a la operación de la planta. Previamente, los alumnos confeccionaron un manual preliminar de operación de la instalación y se procedió a la ejecución de pruebas de estanqueidad y fugas. Estas pruebas consistieron en el llenado de los depósitos e intercambiadores y en la circulación de agua mediante el sistema de bombeo por todas las líneas de la instalación, apareciendo diversos problemas de fugas y mal conexionado de líneas e instrumentación.

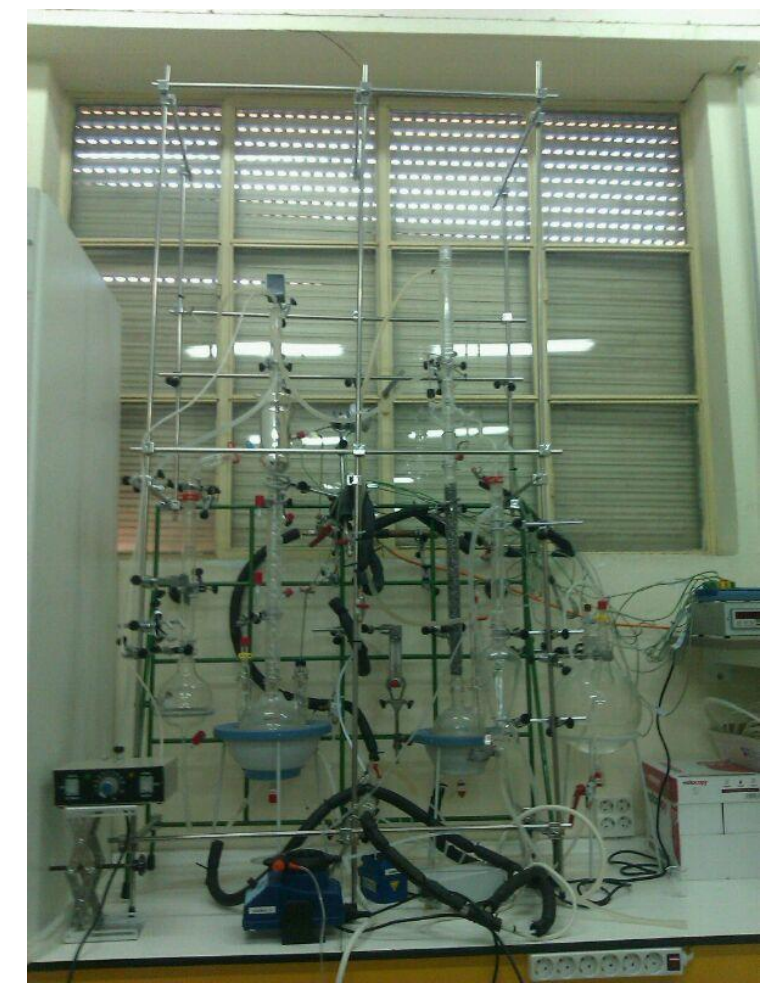

Fig. 2: Unidad de Destilación 
Una vez garantizada la operatividad de la planta, se llevo a cabo la puesta en marcha de la misma. La instalación fue operada durante 1 hora en el punto de diseño (Tabla 5). En estas condiciones, fue posible validar la certificación de la instalación, obteniendo un condesado con una composición en peso promedio del $95 \% \pm 1 \%$ de etanol en agua. La instalación permite obtener el máximo grado de separación posible de la mezcla mediante destilación simple, correspondiente al punto azeotrópico de la mezcla. La sesión de operación permitió actualizar y corregir los manuales preliminares, permitiendo cuantificar los intervalos de operación de las variables manipulables (Tabla 5).

El bloque III concluyó con el entregable 3, considerado como el informe final del proyecto para su evaluación y calificación. El documento definitivo presenta un nivel técnico elevado y es considerado apto para la manipulación y ejecución de prácticas en otras áreas del departamento que se estimen oportunas.

Tabla 5: Intervalos de operación

\begin{tabular}{|l|c|c|c|c|}
\hline \multirow{2}{*}{ Variable } & \multirow{2}{*}{ Unidad } & \multirow{2}{*}{ Punto de Consigna } & \multicolumn{2}{c|}{ Rango de Operación } \\
\cline { 4 - 5 } & & & MIN & MAX \\
\hline Composición alimentación & $\%$ molar en etanol & 30 & 10 & 70 \\
\hline Caudal alimentación & $\mathrm{L} / \mathrm{h}$ & 3 & 1 & 5 \\
\hline Temperatura alimentación & ${ }^{\circ} \mathrm{C}$ & 85 & 80 & 90 \\
\hline Posición de entrada & - & Intermedia & Inferior & Superior \\
\hline Razón de reflujo & - & 1 & 0,5 & 3 \\
\hline Nivel hervidor & - & Medio & Bajo & Alto \\
\hline
\end{tabular}

Para concretar los resultados de satisfacción de los alumnos con esta metodología se hace uso de la encuesta oficial de calidad de enseñanza que anualmente realiza la Universidad de Sevilla en esta asignatura. El cuestionario está compuesto por un total de 18 preguntas donde se evalúa tanto la acción de los profesores como la metodología, estructura y organización de la asignatura. Los resultados individuales sobre cada cuestión han mostrado valores superiores a 4 puntos (sobre 5) en todas ellas. En particular, valoraron con una calificación de 4,2 puntos la organización de la asignatura, con 4,6 puntos el fomento del clima de trabajo y los medios empleados para ello y con 4,8 puntos la consecución de los objetivos de la asignatura mediante la docencia propuesta. La metodología docente evaluada es la expuesta en este trabajo. Los resultados muestran que el grado de satisfacción de los alumnos es muy elevado, 4,8 puntos sobre 5 .

\section{CONCLUSIONES}

El grupo de profesores autores de este trabajo han propuesto y puesto en práctica una nueva metodología de aprendizaje basado en problemas en una asignatura de carácter experimental. El proyecto docente ha comprendido los trabajos necesarios para realizar el montaje de una instalación de destilación. La intención es complementar la metodología docente actual, bajo las directrices expuestas por el EEES, con su aplicación al diseño y montaje de una planta experimental a escala laboratorio que permita a los alumnos el desarrollo de habilidades necesarias para la ejecución profesional de proyectos de Ingeniería de procesos e Ingeniería Química.

El trabajo ha sido realizado por alumnos de último curso de la titulación de Ingeniería Química en la Escuela Técnica Superior de Ingeniería de Sevilla durante 10 sesiones prácticas. Se han ejecutado todas las tareas necesarias para llevar a cabo el proyecto: diseño conceptual e ingeniería básica, gestión de compras, montaje mecánico y eléctrico y puesta en marcha y operación en las instalaciones del departamento. Como resultado de este trabajo, se ha certificado la instalación de una unidad de destilación, siendo posible su uso en asignaturas de experimentación en ingeniería química.

El resultado del proyecto propuesto ha sido satisfactorio dado que los principales objetivos de la propuesta han sido cumplidos. Los alumnos han participado muy activamente, con un alto nivel de motivación e implicación. La nueva metodología ha permitido trabajar todas las competencias adscritas a la asignatura de 'Experimentación en Planta Piloto', siendo el grado de satisfacción del alumnado de 4,8 puntos sobre 5. Se propone ampliar la aplicación de esta metodología en próximos cursos para disponer de una mayor generalidad a la hora de evaluar los resultados obtenidos.

Por lo tanto, esta metodología se presenta como una herramienta muy útil para su aplicación en asignaturas de experimentación en ingeniería química, contribuyendo a una enseñanza de calidad en el ámbito de la ingeniería y cumpliendo con las exigencias docentes marcadas por EEES. 


\section{AGRADECIMIENTOS}

La financiación de la nueva instalación ha correspondido en un 60\% al Departamento de Ingeniería Química y Ambiental y en un $40 \%$ al Instituto de Ciencias de la Educación de la Universidad de Sevilla, mediante la concesión de ayuda para proyectos de innovación docente. Los profesores responsables de esta actividad docente quieren expresar su agradecimiento a José Antonio Camino y a José Antonio Bautista por su colaboración profesional y desinteresada en el montaje de la instalación.

\section{REFERENCIAS}

Ayuga Téllez, E., González García, C. y Grande Ortiz, M. A., Análisis de competencias en el Grado de Ingeniería Forestal para su adaptación al Espacio Europeo de Educación Superior, Form. Univ.: 3(3), 3-14 (2010).

Branda, L.A., El aprendizaje basado en problemas. De herejía artificial a res popularis, Educación Médica: 12(1),11-23 (2009).

Benítez, A. A. y García, M. L., Un primer acercamiento al docente frente a una metodología basada en proyectos, Form. Univ.: 6(1), 21-28 (2013).

Bogumil, F. y Abad, F., Mejoras del aprendizaje y del rendimiento de grupos reducidos de estudiantes en una asignatura de proyectos de ingeniería, Form. Univ.: 1(3), 13-20 (2008).

De la Iglesia, M. I., La identificación de factores en el desarrollo de competencias de los estudiantes universitarios. Un estudio exploratorio, Revista Complutense de Educación: 23(1), 207-240 (2012).

De la Iglesia, M. I., Adecuación del grado de desarrollo de la formación en competencias a la necesidad en el entorno laboral, según la opinión de los estudiantes, Revista Complutense de Educación: 22(1), 71-92 (2011).

De Mesa, J.M., Castañeda, R.J., Sánchez, M. y Mayor, C. Programa de Equipos Docentes para la Formación de Profesores Noveles, Cursos 2004-2005 a 2007-2008, 1a edición, volumen 19, Instituto de Ciencias de la Educación, Sevilla, España (2011).

Jiménez, L., Ramos, F. J. y Ávila, M., Las Universidades españolas y EEES: Un estudio sobre los títulos de grado de maestro en educación primaria, Form. Univ.: 5(1), 3-14 (2012).

Latasa, I., Lozano, P. y Ocerinjauregi, N., Aprendizaje basado en problemas en currículos tradicionales: Beneficios e inconvenientes, Form. Univ.: 5(5), 15-26 (2012).

Ley Orgánica 4/2007, de 12 de abril, de Modificación de la Ley Orgánica de Universidades (LOMLOU) (BOE 89/2007 de 13 de abril de 2007).

Martín, B., Manual de Tuberías, 1ํㅡㄹ edición, Editorial Bernardo Martín Hernández, Bilbao, España (1994).

McCabe, W. L., Smith, J. C. y Harriott, P., Operaciones básicas de ingeniería química, 7ª edición, McGrawHill, Madrid, España (2007).

Morales, P. y Fitzgerald, L., Aprendizaje basado en problemas Problem-based learning, Theoria: 13(1), 145157 (2004).

Pérez-Cedrán, J. C., Lorenzo-Fernández, M. y Cabezas Flores, J. A., Diseño a rigidez de arboles escalonados mediante mathematica®: Un trabajo no presencial en el ámbito del EEES, Form. Uni.: 6 (2), 15-22 (2013).

Perry, R.H., Manual del Ingeniero Químico, 8ª edición, McGraw-Hill, Madrid, España (2007).

Ribeiro, J. C., The Pros and Cons of Problem-Based Learning from the Teacher's Standpoint, Journal of University Teaching \& Learning Practice: 8 (1), Art. 4 (2011).

Rué, J., Enseñar en la Universidad. El EEES como reto para la Educación Superior, $1^{\text {a }}$ edición, Narcea, Madrid, España (2007). 
